\title{
Self-Reinforcing Spatial Patterns Enslave Evolution in a Host-Parasitoid System
}

\author{
Nicholas J. Savill* $*$, Pejman Rohani $\dagger$ and Paulien Hogeweg* \\ * Theoretical Biology and Bioinformatics, Utrecht University, Padualaan 8, 3584 CH Utrecht, \\ The Netherlands; and the $\uparrow$ Department of Zoology, Cambridge University, Downing Street, \\ Cambridge CB2 3EJ, U.K.
}

(Received on 10 January 1997, Accepted in revised form on 19 March 1997)

\begin{abstract}
Spatially structured models of host-parasitoid interactions exhibit self-structuring into spatial patterns such as spiral waves and turbulence. We discuss the consequences of these patterns in an eco-evolutionary model of host-parasitoid interactions with evolution of the parasitoids' ability to disperse towards dense populations of hosts (termed the aggregation strength). It turns out that the direction of, and the time-scale over which the evolutionary selection pressure acts depends on the type of spatial pattern a parasitoid finds itself in. Evolution tends to reinforce the existence of the prevalent local pattern. Moreover, there is also competition between the patterns that ultimately determines the eco-evolutionary attractor. It is the interaction between multiple processes across spatial and temporal scales that leads to the rich meso-scale behaviour. Predicting the evolutionary outcome from statistical measures and subprocesses is shown to give incorrect and conflicting answers. Comparison with the behaviours of the complex Ginzburg-Landau equation shows striking similarities on which we comment.
\end{abstract}

(C) 1997 Academic Press Limited

\section{Introduction}

Localised nonlinear interactions between entities that have limited dispersal rates are likely to form spatial patterns through self-structuring. It is of interest to ask how these spatial patterns feedback on and influence the behaviour of the entities. Recently, much work has been done on this. In particular Hassell et al. (1991) and Comins et al. (1992), studied a spatially structured model of localised hostparasitoid interactions described by the NicholsonBailey equations (Nicholson \& Bailey, 1935) with inter-generation dispersal. They found that persistence of the metapopulation is facilitated by self-structuring into large (meso) scale spatial patterns (i.e., spiral waves and turbulence). Rohani \& Miramontes (1995) extended the model by including parasitoid aggregation. They found it to increase the

\$ Author to whom correspondence should be addressed. E-mail: njs@binf.biol.ruu.nl. domain of the parameter space in which spirals are observed. Further, for given host and parasitoid dispersal rates, there was a specific level of aggregation for which the average host density was a minimum. They proposed that, at this aggregation strength, parasitoids achieve their optimal overall searching strategy. They also remarked that, a parasitoid might be expected to evolve to such an aggregation strength.

As well as having implications for ecological dynamics, spatial pattern formation also has marked and counterintuitive effects for evolutionary dynamics (Boerlijst \& Hogeweg, 1991a, b; Boerlijst et al., 1993; Hogeweg, 1994; Lindgren \& Nordahl, 1994; Claessen \& de Roos, 1995; Keeling \& Rand, 1995; Rand et al., 1995; Savill \& Hogeweg, 1997). In invasion experiments of non-evolving parasitoids Boerlijst et al. (1993) have shown the counterintuitive result that spirals containing parasitoids with low attack rates out-compete spirals containing parasitoids with high attack rates. This is due to the fact

(C) 1997 Academic Press Limited 
that the spiral rotation speed depends on the attack rate of the parasitoids. The lower the parasitoid attack rate the faster a spiral rotates. It is well known that faster rotating spirals can annihilate slower rotating spirals. They concluded that selection shifts from the level of the individuals to the level of the spirals.

In this paper we study the consequences of allowing the aggregation strength of parasitoids of the Rohani \& Miramontes (1995) model to mutate and evolve. This goes beyond the earlier work of Boerlijst et al. (1993) by not separating ecological and evolutionary times-scales a priori. This leads to the consideration of quasi-species instead of monomorphic species and the distribution of the variants in the spatial patterns. Moreover, it enables us to study not only the effect of established (stable) spatial patterns on the direction of the selection, but also the selection of the spatial patterns due to evolution of the "inhabitants".

\section{The Model}

The model of Rohani \& Miramontes (1995) is a spatial extension of the Nicholson-Bailey system of equations, incorporating parasitoid aggregation. We have extended this further to include mutation of the aggregation strength.

Consider a spatially structured environment, consisting of a square lattice of patches. In each generation, a fraction $\left(\alpha_{H}\right)$ of the hosts in a patch disperses uniformly into the neighbouring eight patches. The host density in a patch is then given by:

$$
H^{\prime}=\left(1-\alpha_{H}\right) H+\frac{\alpha_{H}}{8} \sum_{j=1}^{8} H_{j}
$$

where $H$ is the pre-dispersal host density in a patch and $H_{j}$ the pre-dispersal host density in a neighbouring patch $j$.

Once hosts have dispersed, a fraction $\left(\alpha_{P}\right)$ of the parasitoids disperse and aggregate. The strength of aggregation is given by the index $\mu$ as defined by Hassell \& May (1973):

$$
\beta(i)_{A \rightarrow B}=\kappa\left(\frac{H_{B}^{\prime}}{H_{\mathrm{tot}}^{\prime}}\right)^{\mu(i)}
$$

where $\beta(i)_{A \rightarrow B}$ is the proportion of the parasitoid type $i$, aggregating from a patch $A$ in the centre of a nine patch square to a neighbouring patch $B, \kappa$ is a normalisation coefficient such that all $\beta$ 's for a single parasitoid type sum to one and $H_{\text {tot }}^{\prime}$ is the total host density in the patch $A$ and its neighbouring eight patches. For $\mu=0$, parasitoids disperse uniformly into all patches, for $\mu>0$ parasitoids disperse preferentially to high host density patches.

We want to examine the evolution of the aggregation strength, $\mu$. In simulations we have 20 different values of $\mu$. Each parasitoid type $i$ $(=1, \ldots, 20)$ has an aggregation strength of $\mu(\mathrm{i})=i / 10$ (i.e., $\mu=0.1, \ldots, 2.0$ ).

The density of parasitoid type $i$, after dispersal, in a patch $A$, is given by:

$$
P_{i}^{\prime}=\left(1-\alpha_{P}\right) P_{i}+\alpha_{P} \sum_{B=1}^{9} P_{i, B} \beta(i)_{B \rightarrow A}
$$

where $P_{i}$ is the pre-dispersal parasitoid density of type $i$, in a patch and $P_{i, B}$ the pre-dispersal parasitoid density of type $i$ in a neighbouring patch $B$.

The number of viable eggs laid by parasitoid type $i$, in a patch is calculated using the Nicholson-Bailey model:

$$
E_{i}=b H^{\prime}\left(1-e^{-a P_{t o t}^{\prime}}\right) f_{i}
$$

where $b$ is the average number of viable eggs laid by a parasitoid on a single host, $a$ is the per capita parasitoid attack rate, $P_{\text {tot }}^{\prime}$ is the total parasitoid density in that patch and $f_{i}$ is the proportion of hosts parasitised by parasitoid type $i$. On the assumption that a host is parasitised once $f_{i}$ equals $P_{i}^{\prime} / P_{t o t}^{\prime}$.

The host density after parasitism and reproduction and the parasitoid density after mutation and maturation is then given by:

$$
\begin{gathered}
H_{t+1}=\lambda H^{\prime} e^{-a P_{t o t}^{\prime}} \\
P_{i, t+1}=(1-\eta) E_{i}+\frac{\eta}{2}\left(E_{i-1}+E_{i+1}\right)
\end{gathered}
$$

where $\lambda$ is the host reproductive rate and $\eta$ the probability of mutation. Note that type $i=1$ only mutates to $i=2$ and type $i=20$ only mutates to $i=19$.

In order to gain insight into how parasitoid aggregation affected the system's behaviour Rohani \& Miramontes (1995) chose specific dispersal rates for the host and parasitoids $\left(\alpha_{H}=0.2\right.$ and $\left.\alpha_{P}=0.9\right)$. They characterised the patterns for different $\mu$ values. For $\mu<0.4$ there are chaotic spirals. Chaotic spirals are small, short-lived and tend to migrate over short distances. There is continuous formation and annihilation of these spirals. For $0.4 \leqslant \mu \leqslant 1$ spirals become stable and coexistence with turbulence is possible for very long periods. Stable spirals are larger than their chaotic counterparts, they do not migrate and are not annihilated. For $\mu>1$ only turbulence is possible, spirals cannot form. They did note that 


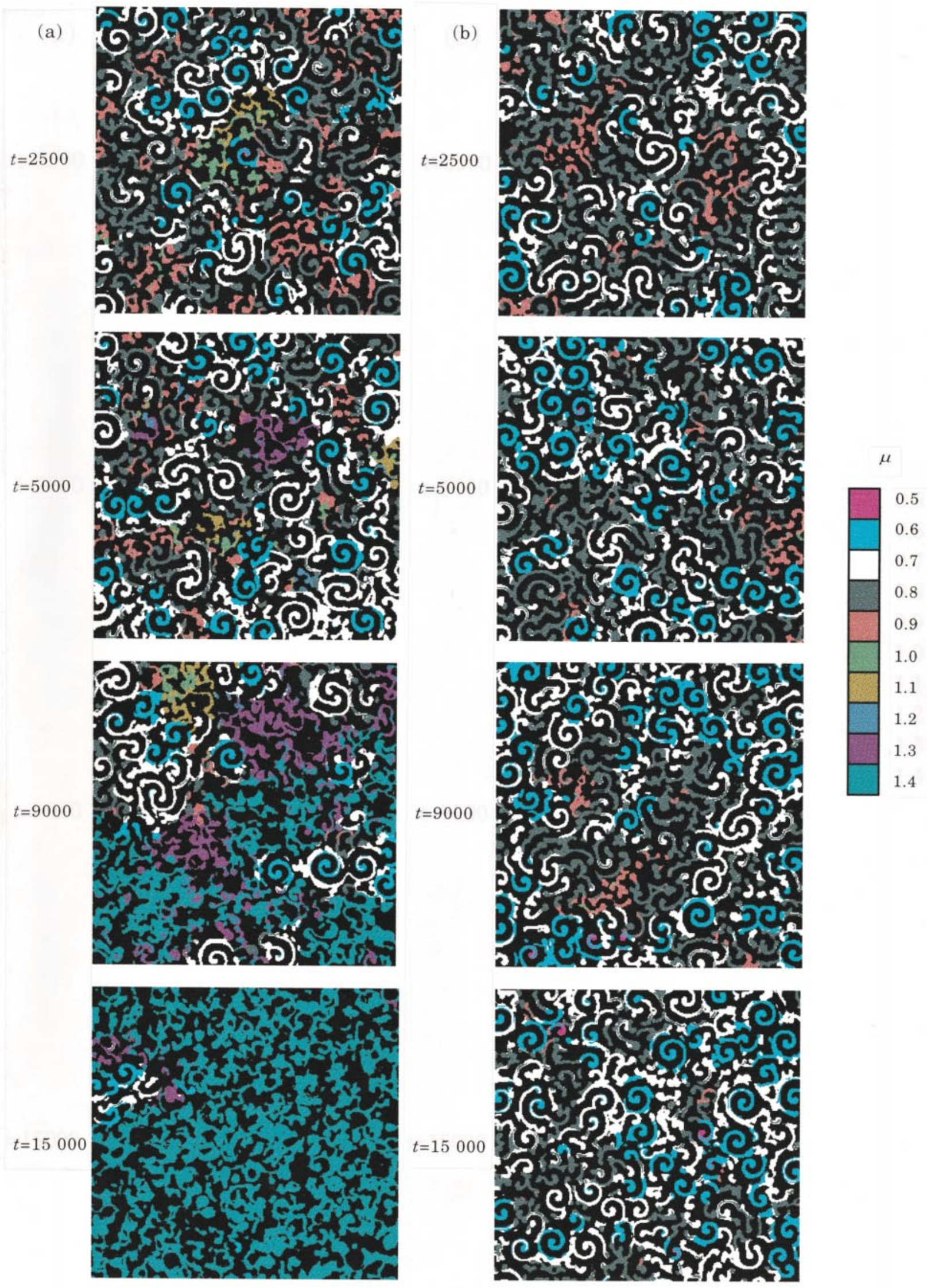

FIG. 1. Snapshots of typical simulations at several generations. The lattice size is $300 \times 300$. The colour of a patch represents the parasitoid type with the highest density in that patch (hosts not shown). If the density of the parasitoid type with the highest density is less than 1 the patch is coloured black to better observe the spatial patterns. Only parasitoids with $0.5 \leqslant \mu \leqslant 1.4$ are observed. Parasitoids with other aggregation strengths exist but hardly ever with the highest density in a patch. Initial and boundary conditions are identical in both simulations. (a) $\alpha_{H}=0.2$. There is a long transient where spirals and high $\mu$ turbulence coexist. However, turbulence, composed of a range of parasitoid types from 1 to 1.8 with $\mu_{e}=1.4$, finally outcompete the spirals. (b) $\alpha_{H}=0.25$. Only spirals and low $\mu$ turbulence exist. High aggregation strengths cannot invade and out-compete spirals. Other parameters are $\alpha_{P}=0.9, \eta=0.001, \lambda=2, b=1$ and $a=0.05$. 
changes in the Nicholson-Bailey parameters only gave quantitative changes to their results. The qualitative features remained. The same also applies in our model. In the simulations discussed in the next section, we keep all parameters constant except $\alpha_{H}$ which takes one of two values, either 0.2 or 0.25 . These values were chosen because they cause the system to settle onto different eco-evolutionary attractors. This will help us in understanding how the observed multi-scale complex processes interact.

The other parameter values are $\alpha_{P}=0.9, \eta=0.001$, $\lambda=2, b=1$ and $\mathrm{a}=0.05$. Two lattice sizes were used $100 \times 100$ and $300 \times 300$ with no difference in the results. Simulations in this paper have a lattice size of $300 \times 300$. There are zero boundary conditions. The initial condition has 90 patches seeded with host and parasitoid densities of 200 and 100 respectively, all other patches are empty. The initial parasitoids have an aggregation strength of $\mu=0.1$.

\section{Results: Selection Pressures and Multiple Scales}

Over many simulations we have found that the system behaviours are independent of the initial and boundary conditions. Figure 1 shows typical simulations for (a) $\alpha_{H}=0.2$ and (b) $\alpha_{H}=0.25$ over 15000 generations. Figure 2 shows how the parasitoid densities change over time.

For $\alpha_{H}=0.2$ there is a long period of coexistence of spirals and turbulence and hence parasitoids with low $\mu(\mu \leqslant 1)$ and high $\mu(\mu>1)$ aggregation strengths respectively. After approximately 10000 generations, the turbulence remains the only spatial pattern in the system. The final stable state is a quasi-species of parasitoids centered around $\mu=1.4$. Mutation keeps the other types, $1.1 \leqslant \mu \leqslant 1.7$, within the system. If the mutation rate is lowered by three orders of magnitude to $\eta=10^{-6}$, the length of time of coexistence of spirals and high $\mu$ turbulence approximately doubles, no other changes are observed. This state is an ESS (Maynard-Smith, 1982), rare parasitoids with much higher and lower aggregation strengths than the quasi-species cannot invade.

For $\alpha_{H}=0.25$ high $\mu$ turbulence does not form and spirals remain the only spatial patterns. Again a quasi-species of parasitoids exists with $0.4 \leqslant \mu \leqslant 1$ with only very small amounts of $1.1 \leqslant \mu \leqslant 1.2$. This state is an ESS, rare lower and higher $\mu$ parasitoids cannot invade. However, for this case low $\mu$ parasitoids are not the only ESS, there are two. If the initial parasitoids have a high $\mu$ then the attractor is the same quasi-species as for $\alpha_{H}=0.2$. The lower mutation rate of $10^{-6}$ makes no difference to the
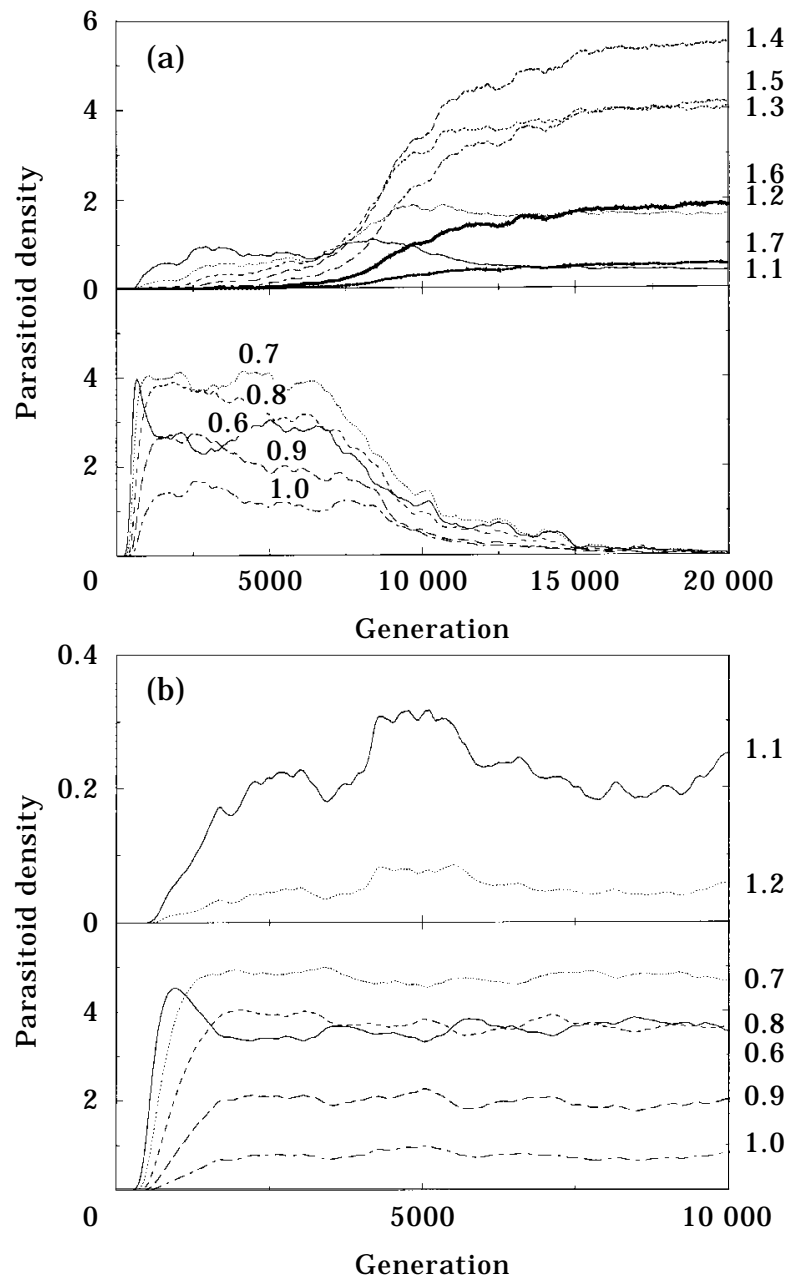

FIG. 2. Parasitoid densities averaged over the whole space for simulations in Fig, 1. For clarity the upper half of the plots show high $\mu$ parasitoids, the lower half the low $\mu$ parasitoids. Each point in a time series is an average over a range of one hundred generations to remove the very large fluctuations. Key: $\mu=0.6$ solid, $\mu=0.7$ dotted, $\mu=0.8$ dashed, $\mu=0.9$ long dashed, $\mu=1$ dot dashed, $\mu=1.1$ solid, $\mu=1.2$ dotted, $\mu=1.3$ dashed, $\mu=1.4$ long dashed, $\mu=1.5$ dot dashed, $\mu=1.6$ thick solid, $\mu=1.7$ thick dotted. (a) $\alpha_{H}=0.2$. (b) $\alpha_{H}=0.25$. Note the scale on the upper plot is much smaller than the lower plot.

dynamics. If mutation is "turned off" for the high $\mu$ quasi-species ESS for both $\alpha_{H}=0.2$ and $\alpha_{H}=0.25$ the quasi-species collapses to a monomorphic species with $\mu=1.4$. However, for the low $\mu$ quasi-species ESS for $\alpha_{H}=0.25$ the quasi-species remains for $0.6 \leqslant \mu \leqslant 1$.

The eco-evolutionary dynamics in the simulations show very long transient behaviour of the order of 1000 s of generations, especially for $\alpha_{H}=0.2$. Studying the processes within these transient periods can help in understanding how the final eco-evolutionary attractors are reached. Moreover, these processes are more than just "a means to an end", they are dynamically complex and interesting in their own 
right. Therefore, in the following discussion we identify and explain, using observations and numerical experiments on the system, how these processes give rise to the transient behaviour. In fact there are too many processes occurring to cover in detail here. Fortunately we can identify from observations a handful of processes that drive, so to say, the eco-evolutionary dynamics towards the attractors. In this section we describe these processes and in the next section we explain why they are so important in determining the eco-evolutionary attractor. The processes that occur are:

(1) spiral formation;

(2) evolution of parasitoids within spirals, occurring over $\sim 100$ s of generations;

(3) evolution of parasitoids within turbulence, occurring over $\sim 100$ s -1000 s of generations;

(4) spiral-spiral and spiral-turbulence competition, occurring over $\sim 1000$ generations.

Note that these processes occur over many temporal and spatial scales and it is this that gives rise to the diverse behaviour exhibited by the system.

\section{SPIRAL FORMATION}

The prominent white and turquoise spirals in Fig. 1 are of the stable variety (i.e., $\mu \geqslant 0.4$ ). Although they are stable they do not persist as in Rohani \& Miramontes (1995) because of the consequences of parasitoid evolution described below. They are surrounded by very small chaotic spirals whose cores are pushed and pulled in space by the influence of the larger spirals. Sometimes the cores are annihilated and sometimes they develop into large stable spirals. Their dynamics are very complex and not really understood.

The mechanism for spiral formation is not known. However, we observe that spirals are only formed in or nearby regions of space where spirals already exist (i.e., where there are many low $\mu$ parasitoids). This can be explained by the fact that spirals "pump" parasitoids into their local environment (Fig. 3). In other words, a spiral seeds its neighbourhood for the formation of new spirals. Spirals cannot form deep within regions of turbulence because no or very few low $\mu$ parasitoids exist in these regions.

\section{EVOLUTION OF PARASITOIDS WITHIN SPIRALS}

The dynamics of spirals (i.e., waves of parasitoids "chasing" waves of hosts away from the core) imply that, on average, more stronger aggregating parasitoids migrate away from the core than weaker aggregating parasitoids. In other words the stronger aggregating parasitoids have a higher efflux from the

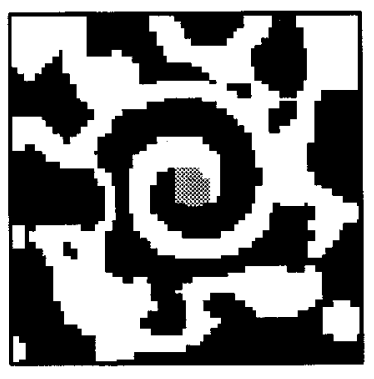

$t=0$

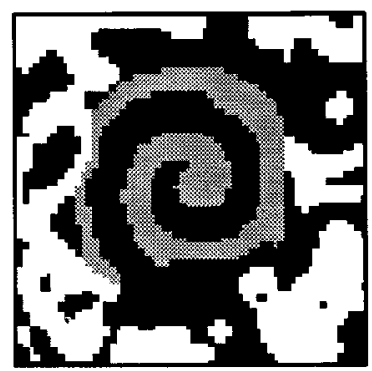

$t=55$

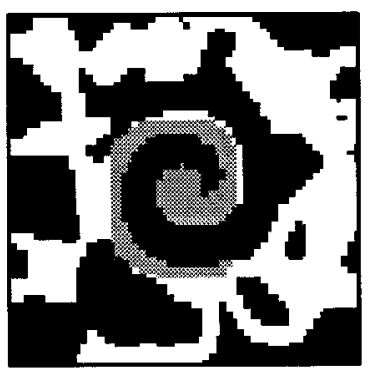

$t=13$

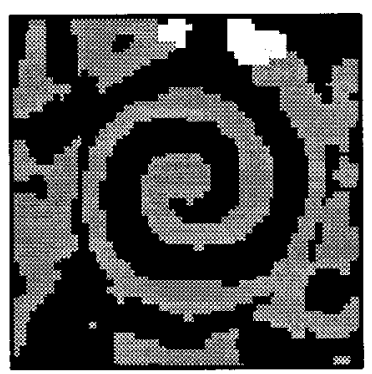

$t=250$
FIG. 3. Simulation with only $\mu=0.7$ parasitoids. The simulation shows two spiral wave behaviours, namely "pumping" of parasitoids from the spiral into the turbulence and the ancestors of descendents within a spiral originate from within the core. At $t=0$ we mark all parasitoids within the core and then follow the descendents of these parasitoids. White: density of descendents from the core $<$ density of descendents from elsewhere, grey: density of descendents from the core $>$ density of descendents from elsewhere, black: density $<1$. At generation 13 the descendents from the core are beginning to take over the spiral. At generation 55 most parasitoids in the spiral are descended from the core. At generation 250 most parasitoids in both the spiral and the turbulence are descended from the spiral core.

core (Fig. 4). Hence over time lower aggregating parasitoids tend to dominate the core (Fig. 5).

In addition to this, most parasitoids within a spiral are descended from parasitoids from within the core (Fig. 3) (Boerlijst \& Hogeweg, 1991b). Therefore, the parasitoids within the whole spiral evolve to lower aggregation strengths. Or, in other words, a spiral reinforces the continuation of itself by causing the evolution of the parasitoid aggregation strength away from high $\mu$ values that would lead toward turbulent dynamics. This process takes on the order of several hundred generations to occur.

\section{EVOLUTION OF PARASITOIDS WITHIN TURBULENCE}

The evolution of the parasitoid aggregation strength in turbulence away from $\mu=1$ to higher values means that turbulence reinforces its ability to exist because spirals can only form where there are many low $\mu$ parasitoids. 


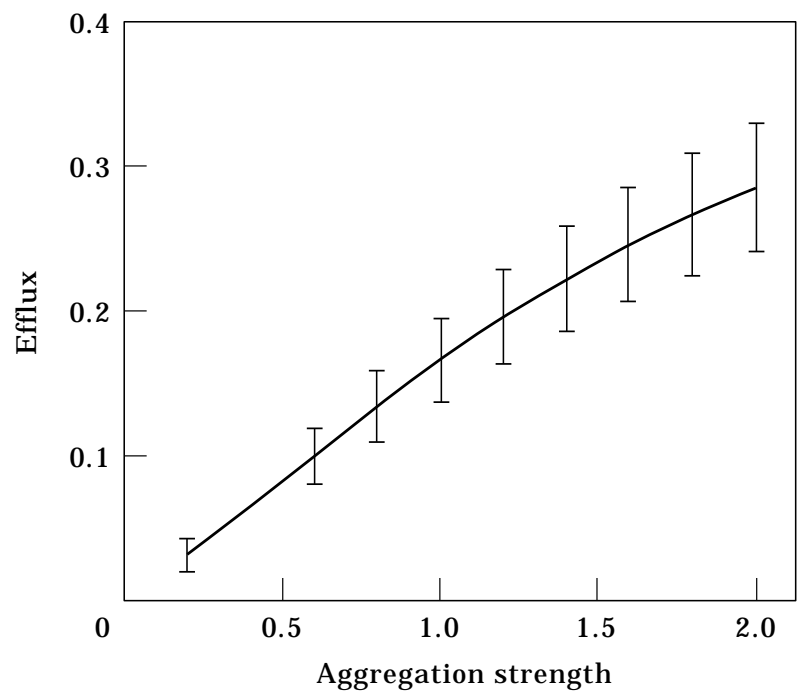

FIG. 4. The efflux of parasitoids from a spiral core due to dispersal and aggregation. Efflux is calculated as the fraction of parasitoids that leave a circle of radius 5 patches centered on the core within one generation (i.e., we do not include the reproduction stage).

The eco-evolutionary attractor is a quasi-species of parasitoids with mean aggregation strength of 1.4. Why this value? We do not have a rigorous mathematical proof but there is some evidence to suggest an answer. Parasitoids that aggregate more strongly into high host density waves produce more

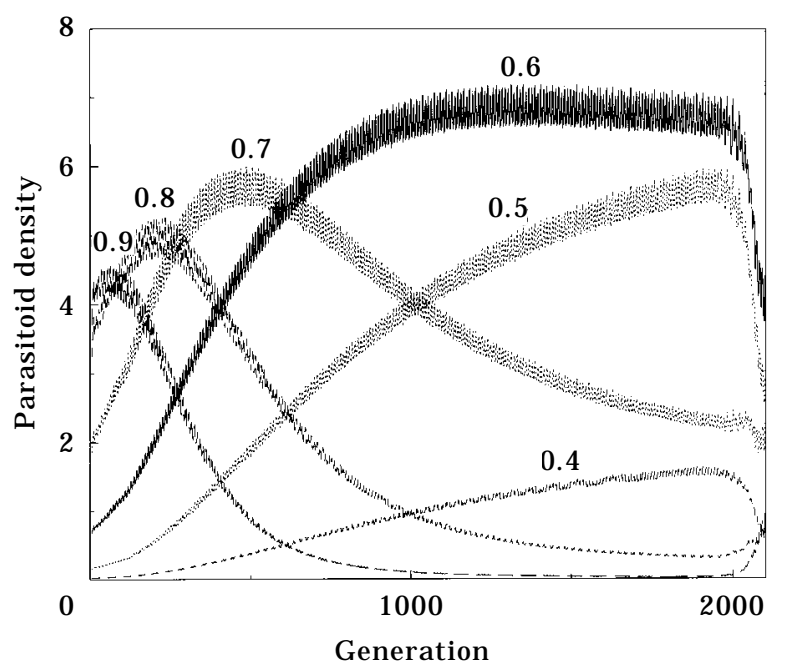

FIG. 5. A typical evolutionary succession of aggregation strengths within a spiral core. Density averaged over a circle, radius 5 patches centered on the spiral core. At generation zero the spiral has just formed and is composed of many parasitoid types with parasitoids with $\mu=0.9$ having the highest density. Over a few hundred generations there is an evolutionary succession towards lower aggregation strengths. Once $\mu=0.6$ parasitoids have the highest density evolution appears to slow down. Around generation 2000 the spiral is annihilated by turbulence before $\mu=0.5$ parasitoids have time to reach the highest density. offspring per individual and therefore have a selective advantage over weaker aggregating parasitoids (Fig. 6). However, the weak aggregating parasitoids produce more offspring per individual in the trough between waves and therefore have a selective advantage over stronger aggregating parasitoids (Fig. 6). This means that when the next host wave arrives there are more weaker aggregating parasitoids than stronger in front of the advancing wave. In other words there are two strategies a parasitoid can adopt: (i) aggregate strongly into the back of a host wave, or (ii) aggregate weakly and wait for the front of the next host wave to arrive. Therefore, below a certain aggregation strength, $\mu_{e}$ it is advantageous to aggregate into high host density waves and above $\mu_{e}$ it is advantageous to remain behind and wait for subsequent waves. These two selection pressures appear to equilibrate at $\mu_{e}=1.4$.

The time-scale for this evolution depends on the local environment. For example, turbulence close to spirals is invaded by many low $\mu$ parasitoids (Fig. 3) and hence evolution to higher aggregation strengths is retarded. Globally however, we see from Fig. 2(a) that $\mu=1.4$ parasitoids are in the majority after roughly 8000 generations.

\section{SPIRAL-SPIRAL AND SPIRAL-TURBULENCE COMPETITION}

Figure 7 shows the time it takes for a spiral to be out-competed by other spirals and turbulence. The length of time taken depends on many factors such as the local neighbourhood of the spiral and the composition of the parasitoid aggregation strengths when it was first formed. The average time is around 1600 generations, approximately three times longer than the time taken for the evolutionary dynamics of the parasitoids within the spirals.

The rotation speed of a spiral depends on the aggregation strengths of the parasitoids it is composed of (Fig. 8). It has been shown both experimentally and theoretically that faster rotating spirals can increase their domain size at the expense of slower rotating spirals (Krinsky \& Agladze, 1983; Maselko \& Showalter, 1991; Winfree, 1991). So spirals composed of higher $\mu$ parasitoids, having a higher rotation speed, out-compete spirals composed of lower $\mu$ parasitoids. In practice, however, only spirals that have a majority of parasitoids with $\mu=0.6$ are outcompeted. This is probably because the evolution of parasitoids within spirals is initially very fast compared with the time it takes for a spiral to be out-competed. Once $\mu=0.6$ parasitoids become the majority type the evolutionary rate slows down considerably (Fig. 5) giving more time for spiral competition to occur. 


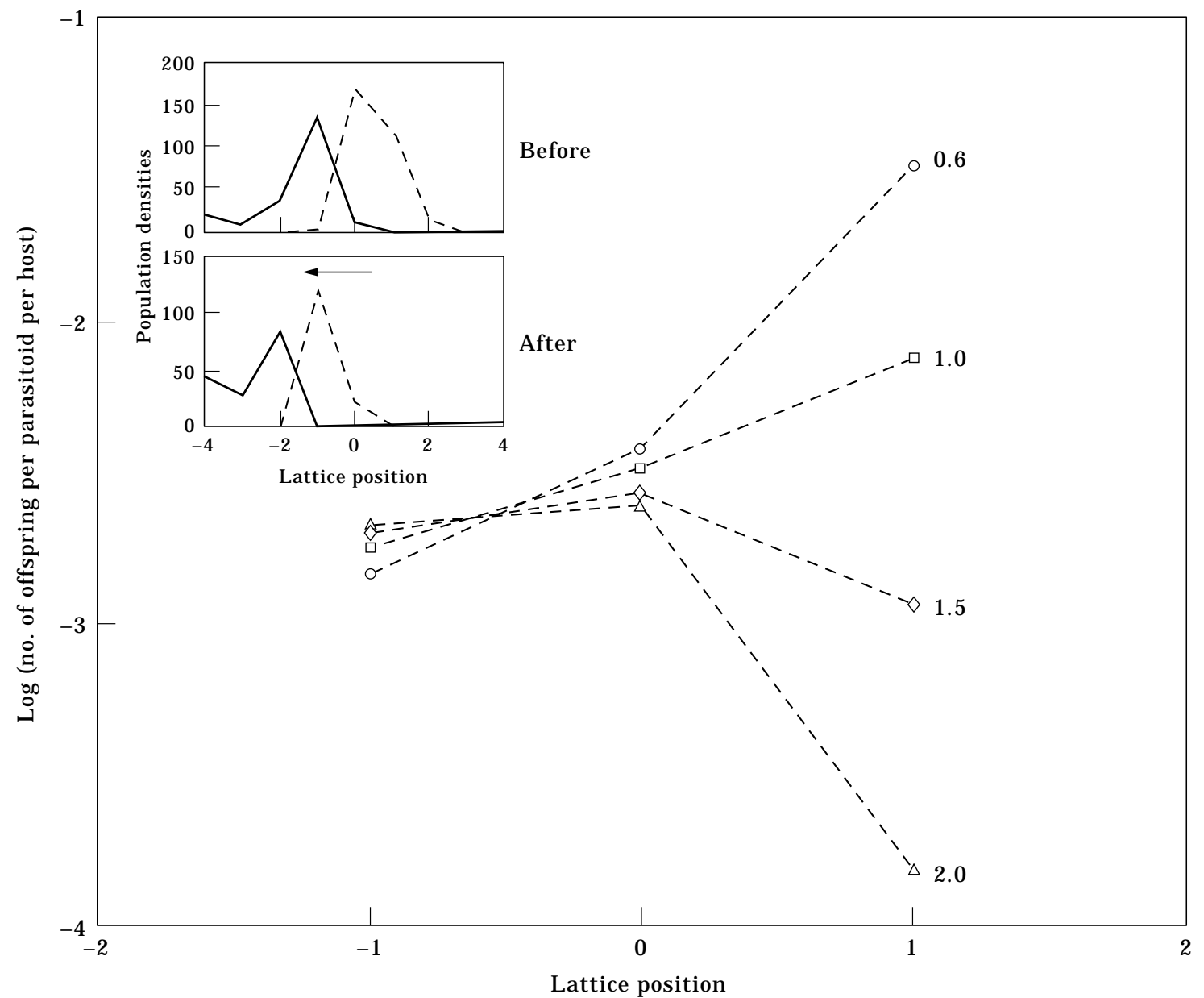

FIG. 6. The inclusive fitness of parasitoids within turbulence. We calculate the number of offspring produced by parasitoids in lattice position 0 after one generation of dispersal and reproduction. The initial total number of parasitoids and hosts is shown in the upper inset. After one generation the parasitoids in lattice position 0 have dispersed to positions -1 and +1 and reproduced. The new host and parasitoid densities are shown in the lower inset. The inclusive fitness for parasitoid aggregation strengths $0.6(\bigcirc), 1(\square), 1.5(\diamond)$ and $2(\triangle)$ are calculated by dividing the number of offspring in the three positions $(-1,0,+1)$ by the initial number of parasitoids in position 0 and by the number of initial hosts in positions $(-1,0,+1)$ respectively. Lower $\mu$ parasitoids produce more offspring per parent per host when dispersing away from host wave and higher $\mu$ parasitoids produce more offspring per parent per host when dispersing into the host wave.

The out-competition of spirals by turbulence is not so well understood. It is probably due to the same mechanism as for spiral-spiral competition, however calculating the average temporal frequency of turbulent dynamics is very difficult as is seen in Fig. 8 (inset). Turbulence also normally out-competes spirals once the majority of the parasitoids have $\mu=0.6$.

\section{What Determines the Eco-evolutionary Attractor?}

Before answering this question it is interesting to see if we could predict the fittest aggregation strength by other means. We do this in order to demonstrate some of the pitfalls one may fall into when predicting the fittest strategy in a spatially extended system.
Firstly, we will consider the proposal by Rohani \& Miramontes (1995) that $\mu_{e}=0.4$. Secondly, we consider the inclusive fitness of parasitoids within spirals and turbulence.

Rohani \& Miramontes (1995) calculated the average host density for different parasitoid aggregation strengths. They found that at $\mu=0.4$, average host densities are at a minimum. They proposed that, at this aggregation strength, parasitoids have their highest attack rate and therefore are the fittest. However, the reason why the average host density is a minimum at $\mu=0.4$ is an artifact of the averaging process. It turns out that at this value there is the maximal number of stable spirals per unit area (results not shown). This implies that the spiral domains are at their smallest. Spirals also have the 


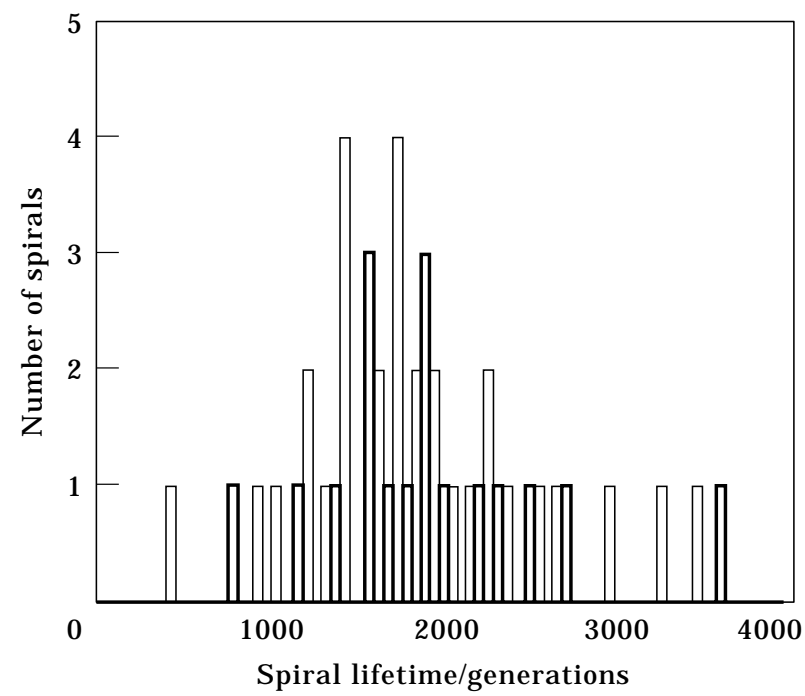

FIG. 7. Histogram of the number of spirals with a certain lifetime calculated for the simulation in Fig. 1a. The lifetime is calculated from when the core first appears until the core is annihilated by other spirals $(\square)$ or turbulence $(\boldsymbol{\square})$. Bin size is 100 generations.

property that their mean values are a minimum in their cores. These two facts explain the minimum at $\mu=0.4$ and has nothing to do with the parasitoid attack rate.

We can calculate the inclusive fitness of a parasitoid at all locations, whether in spirals or turbulent regions, by calculating how many descendents it gives rise to. It turns out that at all locations the higher an individual's aggregation strength is, the more descendants it will have [Fig. 9(a-c)]. Therefore, we might expect evolution to continuously higher aggregation strengths. However, if we calculate the inclusive fitness over a long period of time in a spiral

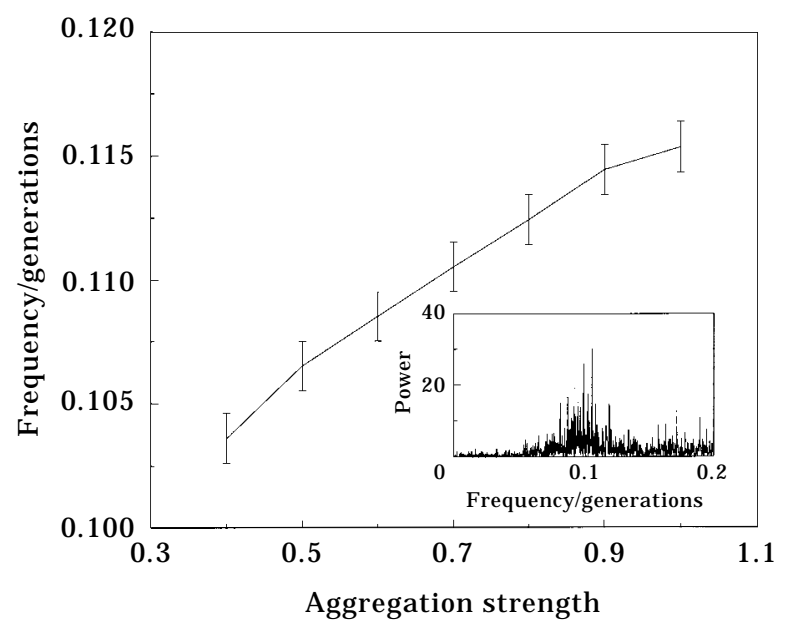

FIG. 8. The rotation frequency of a spiral (in generations ${ }^{-1}$ ) is determined by the aggregation strength of the parasitoids it is composed of. (Inset) the power spectrum of turbulence for $\mu=0.7$ parasitoids shows that there is no well defined single frequency. we see that lower $\mu$ 's become fitter after many generations due to the interaction between short-term local aggregation and long-term mesoscopic spiral dynamics as described in the previous section [Fig. 9(d)].

So the above two predictions about the evolutionary attractor, though at first sight seem plausible, give the wrong answers. Why is this so? Figure 9(d) gives us a clue. To explain how the attractors are reached we must consider all of the processes described in the previous section together and not separately. These processes interact with one another across spatial and temporal scales. A consequence of this is that predicting the attractor may be very difficult.

We can now answer "what determines the eco-evolutionary attractor?". We set up an artificial simulation for $\alpha_{H}=0.25$ in which the left half of the lattice is initialised with low $\mu$ spirals and the right half with high $\mu$ turbulence. Over the course of time it is seen that the spirals out-compete the turbulence (Fig. 10). What is seen is an increase in the number of spirals, that is spirals are being formed faster than they are annihilated. Contrariwise, for $\alpha_{H}=0.2$ spirals are annihilated faster than they can form and hence turbulence wins out. Therefore, we can conclude that the eco-evolutionary attractor is determined by the dynamics on the level of the patterns and not on the level of the individuals. Though, of course, the pattern dynamics are determined by the properties of the individuals.

\section{Discussion and Conclusion}

Pattern formation in population densities can occur via two mechanisms: either externally driven by, for example, gradients in abiotic features, or internally driven by self-structuring. This work hopes to show that pattern formation via self-structuring can have significant consequences for the ecological and evolutionary dynamics of populations. Moreover, eco-evolutionary changes in the system can feedback into the self-structuring mechanisms creating complex dynamics in the patterns themselves.

As an aside, it has been shown that coupled map lattice models that exhibit auto-oscillations, such as this model, show very similar behaviour to the complex Ginzburg-Landau equation (CGLE) (Chaté, 1995). The CGLE is a normal form for the slow amplitude modulations in spatially extended systems near the threshold of a Hopf bifurcation. The qualitative similarities are numerous: coexistence of spirals and turbulence, spiral size varies as a function of the parameters, spiral-spiral interactions, core meandering, bare cores, regular spiral lattices, 

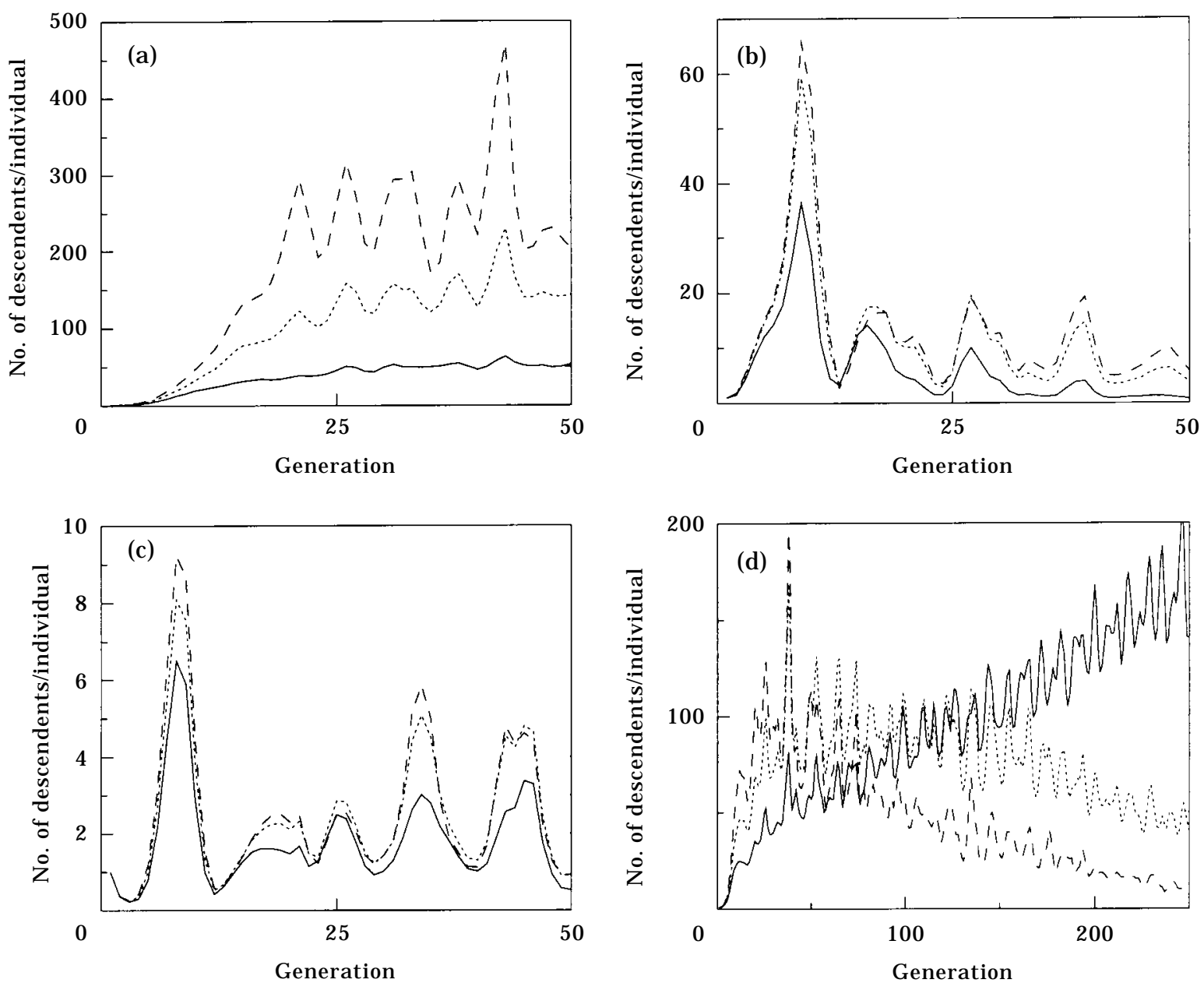

Fig. 9. Inclusive fitness of parasitoid types within spatial patterns. (a) spiral core, (b) spiral arm, (c) turbulence. At generation zero all parasitoids within an area of $5 \times 5$ patches are tagged in each pattern. Over time the number of descendents per tagged individual at generation zero is calculated to give an inclusive fitness for each aggregation strength. In all patterns the higher the aggregation strength the higher the inclusive fitness ( $\mu=0.5$ solid, $\mu=1$ dotted, $\mu=1.5$ dashed). Parasitoids within a spiral core have the highest inclusive fitness of all parasitoids (c.f. $y$-axis scales). (d) shows the number of descendents from a core but plotted over a longer time period. Initially the higher aggregating parasitoids have the highest inclusive fitness but, because of the longer time, the spiral has the opportunity to interact with its environment (in this case turbulence) and the lower aggregating parasitoids have the highest inclusive fitness.

convective instability and spiral stability in the presence of strong environmental noise (results not shown). Understanding the mechanisms that generate the behaviour of the CGLE and comparing it to the behaviour observed in our model has helped us to understand better the processes of the model. In particular we can draw one important conclusion from the comparison. That is that the observed spatial patterns and their interactions are not model dependent. Other work on the ecological effects of spatial pattern formation (e.g., Hassell et al., 1991; Comins et al., 1992; Rohani \& Miramontes, 1995; Ruxton \& Rohani, 1996; Comins \& Hassell, 1996) may also benefit from such a comparison.

For the case of host-parasitoid interactions with evolution of the parasitoid's aggregation strength we have found several interesting results. The direction of the selection on the aggregation strength is opposite in the two spatial patterns. Moreover, the selection pressures are such that they reinforce the persistence of the prevalent patterns.

In Boerlijst et al. (1993) they assumed that the evolutionary time-scale was far longer than the ecological time-scale. This led to the result that a mutant is selected for on the effect it has on the spiral it appears in, either making it rotate faster or slower than before the mutant appeared. In other words, selection shifts from the level of the individual to the level of the spiral. The result is selection for faster rotating spirals and hence for mutants that cause spirals to rotate faster, even though the mutation appears bad for the mutant, i.e., a lower attack rate. 


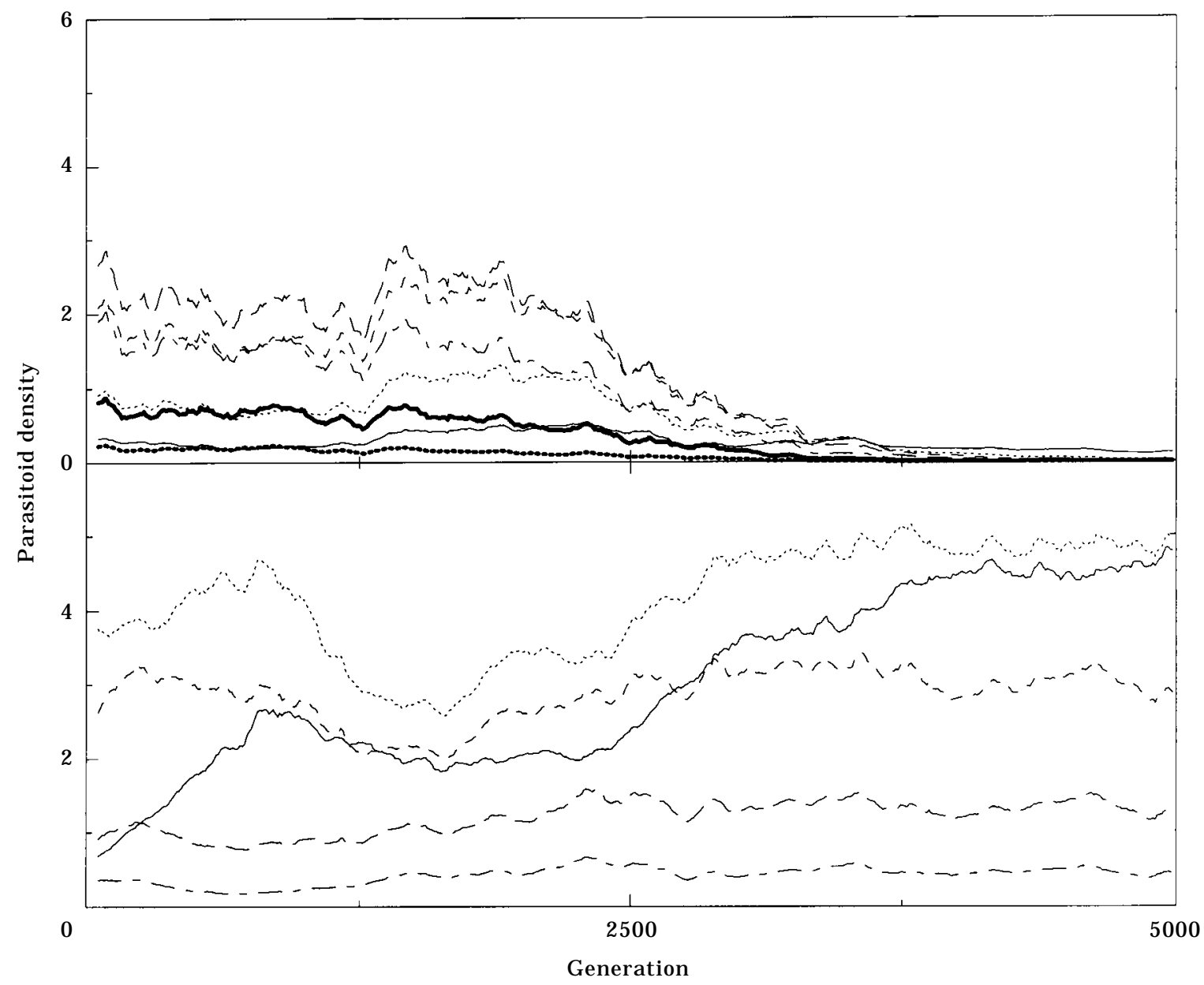

FIG. 10. Parasitoid densities for an artificial simulation for $\alpha_{H}=0.25$. The left half of the lattice is initialised with low $\mu$ spirals and the right half with high $\mu$ turbulence. Each point in a time series is an average over a range of one hundred generations to remove the very large fluctuations. For clarity the upper half of the plot shows high $\mu$ parasitoids, the lower half the low $\mu$ parasitoids. Key: $\mu=0.6$ solid, $\mu=0.7$ dotted, $\mu=0.8$ dashed, $\mu=0.9$ long dashed, $\mu=1$ dot dashed, $\mu=1.1$ solid, $\mu=1.2$ dotted, $\mu=1.3$ dashed, $\mu=1.4$ long dashed, $\mu=1.5$ dot dashed, $\mu=1.6$ thick solid, $\mu=1.7$ thick dotted.

In Boerlijst \& Hogeweg (1995) external spatial gradients were added to an abiotic feature which, for example, influences the death rate of individuals. The gradient causes continuous formation and annihilation of chaotic spirals which can have a positive effect on the elimination of harmful parasites.

In our model we do not assume a priori a separation of the ecological and evolutionary timescales. The result is that the system exhibits multiple selection pressures on different spatial scales (from the individuals to the patterns) and over many temporal scales (from 100 s to 1000 s of generations). For example, a spiral can out-compete slower rotating spirals. But the frequency of rotation changes over time due to the faster selection for parasitoids with lower aggregation strengths within the spiral. These multiple processes occurring over many scales give rise to the long and rich transient behaviour seen in the system. However, the eco-evolutionary attractor is selected for on the basis of the interactions between the patterns.

For $\alpha_{H}=0.2$ parasitoids evolve to an "optimal" quasi-species centered around $\mu=1.4$. Figure 11 shows the variances of the population densities for various values of $\mu$. It clearly shows that the local population dynamics is less stable (i.e., greater chance of extinction) in turbulent patterns than in spiral waves. In other words, parasitoids evolve to an evolutionary stable strategy but at the expense of losing ecological stability. This finding is in qualitative agreement with the conclusions of van Baalen \& Sabelis (1993), who showed that for large regions of the parameter space, evolutionary stable searching strategies of the parasitoid population do not lead to ecological stability.

For $\alpha_{H}=0.25$ observations on the metapopulation 


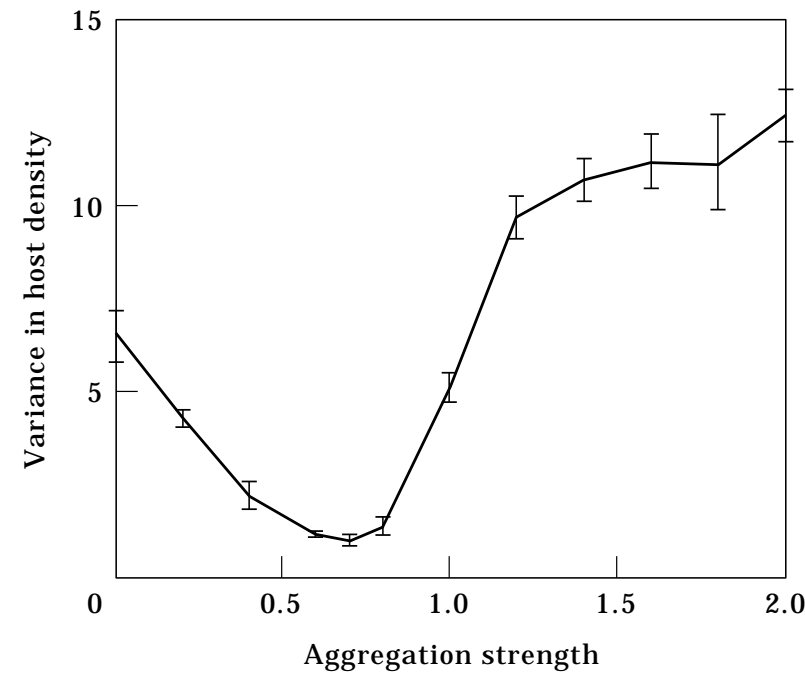

FIG. 11. Variance in the host population densities for different aggregation strengths. A minimum occurs at 0.7 when large stable spirals dominate most of the space. For $\mu>1$ the population dynamics are less stable than weakly aggregating parasitoids. The mean and standard deviations are calculated from 5 time series of 500 generations after initial transients have died.

show a stable quasi-species of low $\mu$ parasitoids. However observing the system on the micro/mesoscales shows that the system is in a continuous flux of spiral formation and annihilation.

We have shown that predicting the eco-evolutionary attractor from statistical measures or subprocesses of the system is fraught with pitfalls and must be handled with care.

Coming onto the more general ideas about the eco-evolutionary consequences of spatial patterns. Clearly, self-structured patterns can play a significant role in the eco-evolutionary dynamics of populations. The entities (host-parasitoid micro-scale populations) that create the patterns (spirals and turbulence) are able to "recognise" the patterns they inhabit. By this we mean there is information encoded in the patterns that directly or indirectly influence the eco-evolutionary dynamics of populations of these entities.

Over and above that, the spatial patterns can interact with one another, for example leading to competition between patterns. The consequence of these pattern-pattern interactions is that the ecoevolutionary dynamics of the micro-scale entities become "slaved" to the dynamics of the meso-scale patterns that they help to create.

N. Savill is supported by the Priority Program Nonlinear Systems of the Netherlands Organization for Scientific
Research. The Dynamics Systems Lab. provided the computer facilities.

\section{REFERENCES}

Boerlijst, M. C. \& Hogeweg, P. (1991a). Self-structuring and selection: spiral waves as a substrate for evolution. Artificial Life II (Langton, C. G., ed.) pp. 255-276. Redwood City, CA: Addison-Wesley.

Boerlisst, M. C. \& Hogeweg, P. (1991b). Spiral wave structure in pre-biotic evolution: hypercycles stable against parasites. Physica D 48, 17-28.

Boerlisst, M. C. \& Hogeweg, P. (1995). Spatial gradients enhance persistence of hypercycles. Physica D 88, 29-39.

Boerlisst, M. C., Lamers, M. E. \& Hogeweg, P. (1993). Evolutionary consequences of spiral waves in a host-parasitoid system. Proc. R. Soc. Lond. B 253, 15-18.

CнатÉ, H. (1995). On the analysis of spatiotemporally chaotic data. Physica D 86, 238-247.

Clatessen, D. \& De Roos, A. M. (1995). Evolution of virulence in a host-pathogen system with local pathogen dispersal. OIKOS 74, $401-413$.

Comins, H. N. \& Hassell, M. P. (1996). Persistence of multispecies host-parasitoid interactions in spatially distributed models with local dispersal. J. theor. Biol. 183, 19-28.

Comins, H. N., Hassell, M. P. \& May, R. M. (1992). The spatial dynamics of host-parasitoid systems. J. anim. Ecol. 61, 735-748.

Hassell, M. P. \& May, R. M. (1973). Stability in insect host-parasite models. J. anim. Ecol. 42, 693-726.

Hassell, M. P., Comins, H. N. \& May, R. M. (1991). Spatial structure and chaos in insect population dynamics. Nature 353, 255-258.

Hogeweg, P. (1994). Multilevel evolution: replicators and the evolution of diversity. Physica D 75, 275-291.

KeELING, M. J. \& RaND, D. A. (1995). A spatial mechanism for the evolution and maintenance of sexual reproduction. OIKOS 74, 414-424.

KRINSKy, V. I. \& AgladZe, K. I. (1983). Interaction of rotating waves in an active chemical medium. Physica D 8, 50-56.

LindGREN, K. \& NoRDAHL, M. G. (1994). Cooperation in artificial ecosystems. Artificial Life 1, 15-37.

Maselko, J. \& Showalter, K. (1991). Chemical waves in inhomogeneous excitable media. Physica D 49, 21-32.

MaynaRd-Smith, J. (1982). Evolution and the Theory of Games. Cambridge: Cambridge University Press.

Nicholson, A. J. \& Bailey, V. A. (1935). The balance of animal populations. Part I. Proc. zool. Soc. Lond. 3, 551-598.

Rand, D. A., Keeling, M. \& Wilson, H. B. (1995). Invasion, stability, and evolution to criticality in spatially extended, artificial host-pathogen ecologies. Proc. R. Soc. Lond. B 259, 55-63.

Rohani, P. \& Miramontes, O. (1995). Host-parasitoid metapopulations: the consequences of parasitoid aggregation on spatial dynamics and searching efficiency. Proc. R. Soc. Lond. B 260, 335-342.

Ruxton, G. D. \& Rohani, P. (1996). The consequences of stochasticity for self-organized spatial dynamics, persistence and coexistence in spatially extended host-parasitoid communities. Proc. R. Soc. Lond. B 263, 625-631.

Savill, N. J. \& Hogeweg, P. (1997). Evolutionary stagnation due to pattern-pattern interactions in a co-evolutionary predatorprey model. Artificial Life 3, 81-100..

van BaAlen, M. \& Sabelis, M. W. (1993). Coevolution of patch selection strategy of predators and prey and the consequences for ecological stability. Am. Nat. 142, 646-670.

WinfreE, A. T. (1991). Alternative stable rotors in an excitable medium. Physica D 49, 125-140. 